\title{
Rescue of common exon skipping mutations in Cystic Fibrosis with modified U1 snRNAs
}

\author{
Stefano Donega' ${ }^{1}$, Malgorzata Rogalska ${ }^{1}$, Giulia Pianigiani ${ }^{1}$, Susana Igreja ${ }^{2}$, Margarida \\ Amaral $^{2}$, and Franco Pagani ${ }^{1}$ \\ ${ }^{1}$ International Centre for Genetic Engineering and Biotechnology \\ ${ }^{2}$ University of Lisbon Faculty of Sciences
}

July 13, 2020

\begin{abstract}
In Cystic Fibrosis $(\mathrm{CF})$, correction of splicing defects represents an interesting therapeutic approach to restore normal CFTR function. In this study, we focused on ten common mutations/variants, $711+3 \mathrm{~A}>\mathrm{G} / \mathrm{C}, 711+5 \mathrm{G}>\mathrm{A}, 1863 \mathrm{C}>\mathrm{T}, 1898+3 \mathrm{~A}>\mathrm{G}$, 2789+5G >A, TG13T3, TG13T5, TG12T5 and 3120G >A that induce skipping of the corresponding CFTR exons 5, 9, 13, 16 and 18. To rescue the splicing defects we tested, in a minigene assay, a panel of modified U1 snRNAs, named Exon Specific U1s (ExSpeU1) that were engineered to bind to intronic sequences downstream of each defective exon. Using this approach, we show that all ten splicing mutations analysed are efficiently corrected by specific ExSpeU1s. Using cDNA-splicing competent minigenes, we also show that the ExspeU1-mediated splicing correction at the RNA level recovered the full-length CFTR protein for $1863 \mathrm{C}>\mathrm{T}, 1898+3 \mathrm{~A}>\mathrm{G}, 2789+5 \mathrm{G}>\mathrm{A}$ variants. In addition, detailed mutagenesis experiments performed on exon 13 led us to identify a novel intronic regulatory element involved in the ExSpeU1-mediated splicing rescue. These results provide a common strategy based on modified U1 snRNAs to correct exon skipping in a group of disease-causing CFTR mutations.
\end{abstract}

\section{Keywords}

Alternative splicing, theranostics, personalized medicine, RNA therapies

\section{Introduction}

Cystic fibrosis (CF; MIM \#219700) is the most common autosomal recessive genetic disease in European descents which affects about 1:2000-4000 new-borns in the US having higher morbidity in some European countries (Burgel et al., 2015; Farrell et al., 2017; Kosorok et al., 1996; Palomaki et al., 2004). The pathology is characterised by a wide spectrum of clinical complications of differing severity varying from classic multiorgan disease to atypical mild monosymptomatic CF forms (Schram, 2012; Zielenski, 2000). In classic CF, lung involvement is the major cause of mortality, dominated by recurrent infections and airway obstruction, finally leading to respiratory failure (Marcorelles et al., 2014). CF is caused by mutation in the Cystic Fibrosis Transmembrane conductance Regulator gene (CFTR, MIM \#602421; GenBank NM 000492.3) (Ontalus et al. 1996; Choi et al. 2001), which codes for a transmembrane $\mathrm{Cl}^{-}$and $\mathrm{HCO}_{3}{ }^{-}$channel. CFTR gene is located on human chromosome 7, contains 27 exons and generates a 6.4 kb mRNA (Gregory et al., 1990) and have been grouped into functional classes which have recently evolved into theratypes (De Boeck \& Amaral, 2016). CFTR disease-causing mutations can affect diverse cellular events including transcription, RNA splicing and protein function (Aissat et al., 2013). In the last years, several studies focusing on how to recover pharmacologically the function of mutated CFTR proteins have identified new drugs that have been approved for the treatment of CF (Hoy, 2019; Strug et al., 2018). These drugs, working as potentiators or correctors recover the defective proteins acting on different CFTR processing steps with a mutation-specific efficacy. This innovative approach has been successfully for developing mutation-oriented 
strategies for personalized medicine. However, this strategy was not specifically conceived for those CFTR mutations that affect precursor mRNA (pre-mRNA) splicing. Among reported mutations, those affecting pre-mRNA splicing represent $12.3 \%$ and many induce exon skipping (Dujardin et al., 2011). Identification of exonic sequences on pre-mRNA requires the splice site consensus sequences as well as a series of exonic or intronic splicing regulatory elements with enhancer or silencer activity (Exonic Splicing Enhancer ESE, Exonic Splicing Silencer ESS, Intronic Splicing Enhancer ISE and Intronic Splicing Silencer ISS). A core of five small nuclear RNPs (snRNP, including U1 snRNP) and several splicing factors along with pre-mRNA secondary structures are important players that act in a coordinated manner to promote the correct exon recognition (Abbink and Berkhout 2008, Zychlinski et al. 2009). Considering the complexity of the splicing process, different types of mutations can induce exon skipping. These mutations are mechanistically different as they can affect consensus sequences at the splice sites, the polypyrimidine tract as well as exonic regulatory elements. As a consequence of this heterogeneity, their rescue may not be an easy task and might require specific approaches. Exon Specific U1s are modified U1 snRNAs that have been shown to rescue different types of exon skipping defects in several diseases. ExSpeU1s have the same composition of normal U1 snRNPs but while U1 snRNPs interact with the 5'splice site (5'ss), ExSpeU1s target intronic sequences by means of their engineered 5' tail (Rogalska et al., 2016). It was previously shown that their binding downstream affected exons corrects aberrant splicing in several cellular (Alanis et al., 2012; Dal Mas, Fortugno, et al., 2015; Nizzardo et al., 2015; Tajnik et al., 2016) and mouse models (Balestra et al. 2014; Dal Mas, Rogalska, et al. 2015; Balestra et al. 2016; Rogalska et al. 2016; Donadon et al. 2018). Strikingly, an ExSpeU1 delivered by Adeno Associated Virus (AAV) resulted to an effective and safe therapy in a Spinal Muscular Atrophy (SMA) mouse model extending the survival from 10 days to ${ }^{\sim} 6$ months (Donadon et al., 2019).

In this study, to prove the potential therapeutic activity of these molecules on CFTR and to establish a useful platform that can be applied to $\mathrm{CF}$, we focussed on ten relatively frequent splicing mutations/variants that cause skipping of corresponding exons 5, 9, 13, 16 and 18. Splicing mutations $711+3 \mathrm{~A}>\mathrm{C} / \mathrm{G}$ and $711+5 \mathrm{G}>\mathrm{A}$ are located in the 5 'ss consensus of exon 5; 1863C $>\mathrm{T}$ (Y577Y) and 1898+3A $>\mathrm{G}$ in an exonic regulatory element and in the 5'ss consensus of exon 13, respectively; 2789+5G $>$ A and 3120G $>$ A are located at the 5'ss consensus of exon 16 and 18 respectively, whereas TG13T3, TG13T5, TG12T5 are variants at the polypyrimidine tract of exon 9 . Using minigene splicing assay we prove that all these mutations can be efficiently corrected by ExSpeU1s in vitro . In addition, detailed analysis of exon 13 suggests that the difficult splicing rescue of this exon might be due to the presence of an Intronic Splicing Silencer (ISS) controlling an unfavourable RNA secondary structure. This result represents the first step towards the development of a personalized approach based on the ExSpeU1 strategy for rescuing CFTR splicing mutations.

\section{Material and methods}

\section{Minigene and Expression vectors design}

CFTR minigenes were created by cloning a sequence composed by an 150 bp intronic regions flanked by the exon examined, respectively exon 5, 9, 13, 16 in the previously described pTB minigene (Pagani et al., 2003) or exon 18 in pFAN minigene (Mattioli et al., 2014). Mutations were introduced in the WT of the respective minigene by site-directed mutagenesis using the Quick-Change Site-directed Mutagenesis Kit II (Agilent, Santa Clara, USA). Exon 13- $\Delta$ minigenes were created by overlapping PCR, using specific oligonucleotides complementary to up-downstream sequences of the deleted region in a pTB-NdeI minigene. ExSpeU1s were created by replacing the sequence between the BclI and BgIII sites with oligonucleotides as previously reported (Pagani et al., 2002). Splicing competent exon 13 minigene and its mutants were made from cDNA cloned in the vector pcDNA 3.1, while for exon 16 we used the described minigenes (Igreja et al., 2015). The identity of minigene constructs was ultimately confirmed through sequencing analysis.

\section{Cell culture, transfection, RNA extraction and CFTR splicing pattern analysis}

HeLa (human cervical carcinoma) cells were grown in DMEM (high glucose, GlutaMAXTM supplement, Gibco) with $10 \%$ foetal bovine serum (FBS, Gibco) at $37^{\circ} \mathrm{C}$ in a humidified incubator in the presence of $5 \% \mathrm{CO} 2$. Cells were plated at a density of $4 * 105$ cells in 6 -well plates at $70 \%$ confluency $24 \mathrm{~h}$ be- 
fore transfection. Transfection was performed using Lipofectamine 2000 (Invitrogen) with CFTR minigenes (500 ng) and Exon Specifics U1 (500 ng, for a total of max $1 \mu \mathrm{g}$ of DNA) and harvested $24 \mathrm{~h}$ later. After $24 \mathrm{~h}$ transfection, RNAs were extracted using Maxwell@ RSC simply RNA Cells Kit in Maxwell Automated RNA extractor (Promega). RT was performed using $1 \mu \mathrm{g}$ of RNA, and cDNA was used in PCR with Taq DNA Polymerase (New England BioLabs). Primers used to amplify CFTR minigenes were oligo-Forward ( $\alpha$ 2,3): 5'-CAACTTCAAGCTCCTAAGCCACTGC-3' and oligo-Reverse (Bra2) 5'TAGGATCCGGTCACCAGGAAGTTGGTTAAATCA-3'. PCR products were separated on a $2 \%$ agarose gel running at $70 \mathrm{mV}$ for 30 minutes and the intensity of the bands were measured with ImageJ software (Rasband, 2012).

\section{Protein isolation and Western blot analysis}

Cells were harvested 48 hours post-transfection, washed twice in 1x phosphate-buffered saline (PBS), and lysed in boiling buffer (1\% [wt/vol] sodium dodecyl sulphate, $1 \mathrm{mM} \mathrm{Na3VO4,} 10 \mathrm{mM}$ Tris, $\mathrm{pH}$ 7.4) containing $0.1 \mathrm{mM}$ phenylmethylsulfonyl fluoride, $10 \mu \mathrm{g} / \mathrm{ml}$ aprotinin, and $5 \mu \mathrm{g} / \mathrm{ml}$ leupeptin for $10 \mathrm{~min}$ at $4^{\circ} \mathrm{C}$. Before separation by sodium dodecyl sulphate-polyacrylamide gel electrophoresis, $40 \mu \mathrm{g}$ of proteins $(20 \mu \mathrm{l}$ volume) were boiled in Laemmli buffer for $5 \mathrm{~min}$. Proteins were then electroblotted onto polyvinyl difluoride membranes (Bio-Rad, Ivry-sur-Seine, France), and nonspecific binding sites were blocked for $2 \mathrm{~h}$ at room temperature by $6 \%(\mathrm{w} / \mathrm{v})$ fat-free milk before an overnight incubation at $4^{\circ} \mathrm{C}$ with specific antibodies for CFTR 570 (1:1000, CFTR Folding Consortium, University of North Carolina,USA) and Tubulin (1:5000). Primary antibodies were detected with horseradish peroxidase-conjugated goat anti-mouse or goat antirabbit IgGs (1:10,000; Jackson ImmunoResearch Laboratories, Villepinte, France) or rabbit anti-goat IgGs (1:5,000; Dako, Glosturp, Denmark). Blots were revealed using an Enhanced Chemioluminescence detection kit (Amersham, Les Ulis, France).

\section{Computational RNA secondary structure prediction and statistical analysis}

Mfold was used for predicting the stability of secondary structures for CFTR exon 13 WT and all the mutants. Free energy of secondary structures was calculated using default settings. Statistical analysis was performed using Microsoft Excel (Microsoft Office Software), GraphPad Prism (GraphPad Software Inc.) and ImageJ. Statistical analyses were done by unpaired Student's T tests $\left({ }^{*} \mathrm{p}<0.05,{ }^{* *} \mathrm{p}<0.01\right.$ and ${ }^{* * *}$ $\mathrm{p}<0.001)$.

\section{Results}

Identification of Exon Specific U1s that correct splicing defects in CFTR exons 5, 9, 13, 16 and 18.

To evaluate the therapeutic potential of ExSpeU1 in CF we focussed on ten splicing defects. Mutations were selected based on their relative high frequency in $\mathrm{CF}$, on exon coverage and on the splicing regulatory elements affected (Table 1). Among those analysed, according to CFTR2 database (www.cftr2.org), 2789+5G $>$ A is the most frequent mutation (1027 alleles reported), followed by $3120 \mathrm{G}>\mathrm{A}, 711+3 \mathrm{~A}>\mathrm{G}$ and $711+5 \mathrm{G}>\mathrm{A}$ (85, 63 and 60 alleles, respectively) and $1898+3 \mathrm{~A}>\mathrm{G}(27$ alleles) while $711+3 \mathrm{~A}>\mathrm{C}$ and $1863 \mathrm{C}>\mathrm{T}(\mathrm{Y} 577 \mathrm{Y})$ are rare mutations. As well, we analysed two polymorphic repeat regions in the 3'- splice site of human CFTR exon 9 affecting the polypyrimidine tract: TG12T5 and TG13T5 associated with atypical CF, and TG13T3 linked to classical CF (Cuppens et al. 1998; Pagani et al. 2000; Pagani et al. 2003; Groman et al. 2004; Du et al. 2014; Boussaroque et al. 2020). Altogether the mutations we have analysed cover five CFTR exons: 5, 9, 13, 16 and 18. Six mutations are located in the 5'ss (donor) consensus, $(711+3 \mathrm{~A}>\mathrm{C} / \mathrm{G}$, $711+5 \mathrm{G}>\mathrm{A}, 1898+3 \mathrm{~A}>\mathrm{G}, 2789+5 \mathrm{G}>\mathrm{A}, 3120 \mathrm{G}>\mathrm{A}$ ), one affects an exonic regulatory element $(1863 \mathrm{C}>\mathrm{T})$ and three variants involve the polypyrimidine tract (TG12T5, TG13T5 and TG13T3). In all mutations, rescue of exon skipping will result in the production of a normal coding CFTR mRNA either because the mutations are intronic or because they are synonymous. To test the splicing rescue activity of ExSpeU1s, we created minigenes with exons and corresponding flanking intronic regions that contain the target wild type (WT) and mutant sequences. CFTR exon 5 minigene experiments showed that, in basal conditions, the WT construct was nearly completely included whereas $711+3 \mathrm{~A}>\mathrm{C}$ and $711+5 \mathrm{G}>\mathrm{A}$ induced severe exon 
5 skipping and $711+3 \mathrm{~A}>\mathrm{G}$ retained some exon inclusion ( $\sim 18 \%$ ) (Fig.1 A, lanes 1, 2, 4 and 3, respectively). We tested four ExSpeU1s binding downstream the 5'ss (U1ex5-9, U1ex5-13, U1ex5-21 and U1ex5-27 bp) (Fig.1A). These ExSpeU1s induced complete rescue of the exon 5 splicing defects; the only exception was represented by U1ex5-21 and U1ex5-27 that partially recovered ( $52 \%)$ the $711+3 \mathrm{~A}>\mathrm{C}$ variant. For exon 9 , we analysed four TG(m)T(n) variants: TG11T7, TG12T5, TG13T5 and TG13T3 along with four ExSpeU1s (U1ex9-3, U1ex9-12, U1ex9-26 and U1ex9-34). These mutations induce different degrees of exon skipping (Fig. 1B) with activation of a cryptic site inside the exon, as previously reported (Ayala et al., 2006; Buratti et al., 2001; Zuccato et al., 2004). In basal condition, TG11T7 showed $\sim 98 \%$ of exon inclusion, TG12T5

61\%, TG13T5 54\% and TG13T3 6\% (Fig. 1B). ExSpeU1s co-transfection totally rescued aberrant splicing in TG13T5 and TG12T5. In TG13T3, splicing rescue was 72 - 84\% for U1ex9-3, U1ex9-12, U1ex926, whereas downstream ExSpeU1s had a lower effect (U1ex9-34 51\%). We then evaluated two exon 13 variants, $1863 \mathrm{C}>\mathrm{T}$ and $1898+3 \mathrm{~A}>\mathrm{G}$ that induce respectively $4 \%$ exon inclusion and complete skipping, respectively (Fig.1 C, lanes 2 and 3). We tested several ExSpeU1 but only one U1ex13-11 was working efficiently by rescuing both variants to WT levels of exon inclusion, suggesting that the CFTR exon 13 context has some peculiarity that restricts the ExSpeU1 splicing capacity. To evaluate $2789+5 \mathrm{G}>\mathrm{A}$ variant in exon 16 we tested five ExSpeU1s (U1ex16(-3), U1ex16-7, U1ex16-10, U1ex16-12 and U1ex16-17) (Fig.1D). In this case, the mutant showed $53 \%$ exon inclusion and all ExSpeU1s were able to completely correct the splicing defect (Fig. 1 D). We next analysed the $3120 \mathrm{G}>\mathrm{A}$ variant in exon 18 . This mutation, located in the last base of the exon, induced complete exon 18 skipping (Fig. 1E, lane 2). The different ExSpeU1 tested were able to efficiently rescue the splicing defect: U1ex18-3 and U18-20 induced $85 \%$ of exon inclusion and U1ex18-10 and U18-13 100\% (Fig. 1 E). Altogether our results have identified active ExSpeU1s able to correct with high efficiency ten splicing defects that affect CFTR exon 5, 9, 13, 16 and 18.

\section{Exon Specific U1s recover CFTR protein levels in exon 13 and 16 mutant minigenes}

To evaluate if the splicing correction mediated by Exon Specific U1 rescues the CFTR protein, we focussed on exon 13 and 16. The WT and mutant CFTR exons were inserted into cDNA splicing competent minigenes that code for the entire CFTR protein in a manner that the exon inclusion mRNA variants will result in the production of a normal CFTR proteins. The minigenes were transfected along with each active ExSpeU1s and CFTR protein analysed by Western blotting. As expected from the severity of the splicing defect (Fig.1C), the two exon 13 mutants, $1898+3 \mathrm{~A}>\mathrm{G}$ and $1863 \mathrm{C}>\mathrm{T}$ (Y577Y) did not show any detectable CFTR mature protein in Western blotting (Fig.2A, lanes 4 and 5). Co-transfection of U1ex13-11 rescued both the exon skipping defects (Fig.1C) with production of the full-length fully-glycosylated 160KD form of CFTR protein (Fig. 2A, lanes 6 and 7). Control full-length CFTR cDNA and a minigene with the WT exon 13 showed the mature CFTR form (Fig.2A lanes 1 and 3, respectively) whereas a minigene without exon 13 did not show any band (Fig. 2A, lane 2). Consistent with a residual splicing activity (Fig.1), the mutation 2789+5G $>$ A mutation in exon 16 showed a reduced amount of the CFTR mature form, compared to the WT minigene (Fig. 2B, compare lanes 1 and 2). Treatment with the three active ExSpeU1s, U1ex16(-3), U1ex16-7 and U1ex16-12) showed a two-fold increase in the amount of mature CFTR protein of $160 \mathrm{kDa}$ (Fig. 2B, lanes 3, 4 and 5).

An intronic regulatory element that overlaps with U1ex13-11 binding site regulates exon 13 splicing and forms a stem-loop RNA secondary structure

Among the CFTR exons evaluated, exon 13 was the most challenging to rescue. In fact, at variance to the other exons, we have identified only one ExSpeU1, U1ex13-11, with a reliable splicing rescue activity on both $1863 \mathrm{C}>\mathrm{T}$ and $1898+3 \mathrm{~A}>\mathrm{G}$ mutations (Fig. 1A). Thus, we decided to explore if there is any specific reason for this apparent lack of efficiency. To this aim we focussed on intronic regulatory elements within the ExSpeU1 binding region performing consecutive intronic deletions in $\mathrm{WT}, 1863 \mathrm{C}>\mathrm{T}$ and $1898+3 \mathrm{~A}>\mathrm{G}$ (Fig. 3A) minigenes. Interestingly, deletion of bases in position 12-15 and 17-23 downstream of the 5'ss, but not in the other positions, improved splicing in both the WT and in the $1863 \mathrm{C}>\mathrm{T}$ contexts (Fig. 3A, lanes 2,3 and 9,10, respectively). These deletions, however, did not affect splicing in the $1898+3 \mathrm{~A}>\mathrm{G}$ minigene. These results indicate that the binding sequence of the active U1ex13-11 (between positions +11 and +26 ) 
overlaps with an Intronic Splicing Silencer (ISS) located between position +11 and +23 . In this context, the strength of the ISS is not sufficient to recover exon 13 skipping caused by the 5'ss mutation. In addition, analysis with RNA-mfold (Wang et al., 2017) showed that the ISS is located within a 7bp-long stem-loop that include the 5'ss (Fig. 3B). This configuration suggests that the peculiar RNA secondary structure where the ISS is located might limit the accessibility of the U1snRNP to the 5'ss, as previously reported in other cases (Buratti \& Baralle, 2004). To clarify the ISS mechanism in detail we performed site directed mutagenesis experiments assisted by RNA secondary structure analysis in the loop (positions +12 and +13 ), in the stem (positions +14 to +19 ) and outside the structure (positions +20 to +23 ). The resulting effect on splicing expressed as changes in percentage of exon inclusion was then related to the predicted effect on the secondary structure expressed as $\Delta \mathrm{G}$, that is the quantity of energy needed to fully break the secondary structure (Fig. $3 \mathrm{D}$ and Table S1). Mutagenesis from the +14 to +19 position are predicted to open the stem of the structure and accordingly in splicing assay they increase exon inclusion (Fig. 3C, lanes 8-18). In contrast, the mutants predicted to close the loop, namely $+13 \mathrm{C},+12 \mathrm{C} 13 \mathrm{C}$ and $+12 \mathrm{~A} 13 \mathrm{C}$, had a negative effect on exon 13 inclusion and induced nearly complete skipping (Fig. 3C, lanes 5, 25, 26). All other mutants in the loop $(+12 \mathrm{~T},+12 \mathrm{~A}$, $+12 \mathrm{C},+13 \mathrm{~T},+13 \mathrm{G}+12 \mathrm{~A} 13 \mathrm{~T},+12 \mathrm{C} 13 \mathrm{G})$ and those outside of the structure $(+20 \mathrm{~A},+20 \mathrm{G},+21 \mathrm{G},+22 \mathrm{~T}$, $+22 \mathrm{~A},+23 \mathrm{G})$, that do not significantly affect the secondary structure, had no significant effect on splicing. Based on the predicted changes in $\Delta \mathrm{G}$ (Table S1) and the resulting change in percentage of exon inclusion we modelled association between the change in stability of the secondary structure and the impact on splicing (Fig. 3D). This analysis showed a clear sigmoidal association between splicing of exon 13 and $\Delta \mathrm{G}$, supporting the hypothesis that the stem-loop structure at CFTR exon 13 5'ss is involved in splicing regulation and that this might restrict the effect of ExSpeU1s to specific intronic binding sites.

\section{Discussion}

In last years, research on CF has been very successful in developing personalised therapies based on the correction of the underlying basic defects (De Boeck \& Amaral, 2016; Fajac \& Wainwright, 2017; Farinha \& Matos, 2016; Paranjape \& Mogayzel, 2018; I. Pranke et al., 2019; Roomans, 2014). This has led to the discovery of drugs that act on specific CFTR protein mutants either as potentiators or correctors (Boyle et al., 2014; Chaudary, 2018; Clancy, 2018; Gees et al., 2018; Gentzsch \& Mall, 2018; Guimbellot et al., 2017; Phuan et al., 2018; Van Goor et al., 2009; Wu et al., 2019; Yeh et al., 2017). However, CF individuals with mutations that disrupt other basic mechanisms, and in particular CFTR RNA processing, cannot benefit of these personalized therapies. In this paper, to provide a useful rescuing approach acting at the RNA level, we focussed on a group of splicing mutations that induce exon skipping. We show here that a strategy based on modified U1 snRNAs, named Exon Specific U1s (ExSpeU1), when applied to ten representative splicing mutations, can correct the resulting exon skipping defects with recovery of the normal CFTR protein. With ten mutations and five exons analysed, our study is the first to show a therapeutic effect of an exon rescue strategy on several splicing mutations and various exons in a single gene. Indeed, in CF a different approach based on Anti Sense Oligonucleotides (ASO) has been tested only in the $2789+5 \mathrm{G}>\mathrm{A}$ splicing mutation (Igreja et al. 2016). In general, this alternative ASO based approach for rescuing exon skipping defects is based on the identification of appropriate splicing regulatory elements and thus more time consuming and expensive and not easy testable on several splicing defects. Similarly, a pharmacological approach to correct splicing requires high-throughput screening platforms and may lack of specificity (Berg et al., 2019; Giuliano et al., 2018; Liang et al., 2017; Merkert et al., 2019; Pereira et al., 2019). The positive effect we observed here on different CFTR mutations, along with those previously reported for other diseases in several cellular and mouse models (Dario Balestra et al., 2020; Dal Mas, Fortugno, et al., 2015; Donadon et al., 2018; Tajnik et al., 2016), indicates a general applicability and translatability of the ExSpeU1 approach in rescuing exon skipping defects. Even if our results show a clear rescue potential of the ExSpeU1 approach in CF, the therapeutic window is restricted to those splicing defects that do not disrupt the invariant AG and GT dinucleotides of the splice site. These more severe cases cause a complete disruption of the splicing process and thus cannot benefit of the ExSpeU1-mediated rescue approach. In CF, we estimate that approximately 40 splicing mutations out of 352 CF-causing variants listed in CFTR2 database could benefit from the ExSpeU1 approach. The specificity and easy tailoring of the ExSpeU1 strategy is mainly related to its engineered 5, 
tail that targets the ExSpeU1 on desired intronic sequences. As we identified for each CFTR exon a panel of active ExSpeU1s (with the notable exception of exon 13), several molecules could be then tested for better efficacy and safety. An important concern for ExSpeU1s, as well as for other splicing correction strategies with ASO or chemical compounds, is their potential off-target effects. Previous studies in cellular and animal models with one ExSpeU1 active on Spinal Muscular Atrophy identified a very limited number of off-targets by RNA-Seq analysis (Dal Mas, Rogalska, et al., 2015; Donadon et al., 2019). Indeed, the availability of cellular systems that overexpress the ExSpeU1s will allow the identification of the most promising and safe molecules active on the different CFTR exons. In CFTR exons 5,9 and 18, the ExSpeU1s tend to reduce their exon rescue efficacy when binding to more downstream intronic positions (Fig.1) and this phenomenon has been also observed previously in other cases (Alanis et al., 2012; Dal Mas, Fortugno, et al., 2015; Tajnik et al., 2016). Thus, the detection of only one ExSpeU1s active on exon 13 mutants was unexpected. We reasoned that the peculiar context of this exon could be involved and indeed we have identified in intron 13 an ISS that, through the formation of a stem-loop RNA secondary structure in the proximity of the 5'ss, has a negative effect on splicing. Similar stem-loop structures are known to modulate the 5'ss accessibility in other exon skipping defects (Buratti \& Baralle, 2004). Thus, we speculate that the overlap between the ISS and the binding region of U1ex13-11 might explain why only this one was active on exon 13 splicing mutants. It is possible that part of the U1ex13-11 efficacy, which is not shared by the adjacent ExSpeU1s, relies on its ISS binding position with consequent opening of the unfavourable RNA secondary structure.

The short ExSpeU1 coding gene ( $650 \mathrm{bp}$ ) can be easily accommodated into Adeno Associated Viruses or Lentiviral Vectors forin vivo gene therapy (Rogalska et al. ,2016; Donadonet al. , 2018) or ex vivo cellular delivery (Dal Mas, Fortugno, et al., 2015; Dal Mas, Rogalska, et al., 2015; Nizzardo et al., 2015). Alternative delivery approaches that could be tested include the de novo chemical RNA synthesis (Ohkubo et al., 2013). The availability of patient-derived cellular models with the splicing mutations in form of nasal epithelial cells (I. M. Pranke et al., 2017) or organoids (Boj et al., 2017; Dekkers et al., 2013, 2016; Sato et al., 2009, 2011) will allow a more direct assessment of the ExSpeU1s strategy on the CFTR functionality and the evaluation of the ExSpeU1 efficacy and safety profile in patient-derived target cells.

\section{Acknowledgements}

F.P. is listed as inventor of the US patent n. 9669109 'A modified human U1snRNA molecule, a gene encoding for the modified human U1snRNA molecule, an expression vector including the gene, and the use thereof in gene therapy of familial dysautonomia and spinal muscular atrophy'. As such the inventors could potentially benefit from any future commercial exploitation of patent rights, including the use of ExspeU1s in $\mathrm{CF}$.

\section{References}

Abbink, T. E. M., \& Berkhout, B. (2008). RNA Structure Modulates Splicing Efficiency at the Human Immunodeficiency Virus Type 1 Major Splice Donor. Journal of Virology , 82 (6), 3090-3098. https://doi.org/10.1128/JVI.01479-07

Aissat, A., de Becdelièvre, A., Golmard, L., Vasseur, C., Costa, C., Chaoui, A., Martin, N., Costes, B., Goossens, M., Girodon, E., Fanen, P., \& Hinzpeter, A. (2013). Combined Computational-Experimental Analyses of CFTR Exon Strength Uncover Predictability of Exon-Skipping Level. Human Mutation, 34 (6), 873-881. https://doi.org/10.1002/humu.22300

Alanis, E. F., Pinotti, M., Mas, A. D., Balestra, D., Cavallari, N., Rogalska, M. E., Bernardi, F., \& Pagani, F. (2012). An exon-specific U1 small nuclear RNA (snRNA) strategy to correct splicing defects. Human Molecular Genetics , 21 (11), 2389-2398. https://doi.org/10.1093/hmg/dds045

Ayala, Y. M., Pagani, F., \& Baralle, F. E. (2006). TDP43 depletion rescues aberrant CFTR exon 9 skipping. FEBS Letters ,580 (5), 1339-1344. https://doi.org/10.1016/j.febslet.2006.01.052

Balestra, D., Faella, A., Margaritis, P., Cavallari, N., Pagani, F., Bernardi, F., Arruda, V. R., \& Pinotti, M. (2014). An engineered U1 small nuclear RNA rescues splicing-defective coagulation F7 gene expression in 
mice. Journal of Thrombosis and Haemostasis ,12 (2), 177-185. https://doi.org/10.1111/jth.12471

Balestra, Dario, Scalet, D., Ferrarese, M., Lombardi, S., Ziliotto, N., Croes, C. C., Petersen, N., Bosma, P., Riccardi, F., Pagani, F., Pinotti, M., \& van de Graaf, S. F. J. (2020). A compensatory U1snRNA partially rescues FAH splicing and protein expression in a splicing-defective mouse model of tyrosinemia type I.International Journal of Molecular Sciences , 21 (6). https://doi.org/10.3390/ijms21062136

Balestra, Dario, Scalet, D., Pagani, F., Rogalska, M. E., Mari, R., Bernardi, F., \& Pinotti, M. (2016). An exon-specific U1snRNA induces a robust factor IX activity in mice expressing multiple human FIX splicing mutants. Molecular Therapy - Nucleic Acids , 5 (10), e370. https://doi.org/10.1038/mtna.2016.77

Berg, A., Hallowell, S., Tibbetts, M., Beasley, C., Brown-Phillips, T., Healy, A., Pustilnik, L., Doyonnas, R., \& Pregel, M. (2019). High-Throughput Surface Liquid Absorption and Secretion Assays to Identify F508del CFTR Correctors Using Patient Primary Airway Epithelial Cultures. SLAS Discovery , 24 (7), 724-737. https://doi.org/10.1177/2472555219849375

Boj, S. F., Vonk, A. M., Statia, M., Su, J., Vries, R. R. G., Beekman, J. M., \& Clevers, H. (2017). Forskolininduced swelling in intestinal organoids: An in vitro assay for assessing drug response in cystic fibrosis patients. Journal of Visualized Experiments, 2017 (120). https://doi.org/10.3791/55159

Boussaroque, A., Audrézet, M. P., Raynal, C., Sermet-Gaudelus, I., Bienvenu, T., Férec, C., Bergougnoux, A., Lopez, M., Scotet, V., Munck, A., \& Girodon, E. (2020). Penetrance is a critical parameter for assessing the disease liability of CFTR variants. Journal of Cystic Fibrosis , 0 (0). https://doi.org/10.1016/j.jcf.2020.03.019

Boyle, M. P., Bell, S. C., Konstan, M. W., McColley, S. A., Rowe, S. M., Rietschel, E., Huang, X., Waltz, D., Patel, N. R., \& Rodman, D. (2014). A CFTR corrector (lumacaftor) and a CFTR potentiator (ivacaftor) for treatment of patients with cystic fibrosis who have a phe508del CFTR mutation: A phase 2 randomised controlled trial. The Lancet Respiratory Medicine , 2 (7), 527-538. https://doi.org/10.1016/S22132600(14)70132-8

Buratti, E., \& Baralle, F. E. (2004). Influence of RNA Secondary Structure on the Pre-mRNA Splicing Process. Molecular and Cellular Biology, 24 (24), 10505-10514. https://doi.org/10.1128/mcb.24.24.1050510514.2004

Buratti, E., Dörk, T., Zuccato, E., Pagani, F., Romano, M., \& Baralle, F. E. (2001). Nuclear factor TDP-43 and SR proteins promote in vitro and in vivo CFTR exon 9 skipping. EMBO Journal , 20 (7), 1774-1784. https://doi.org/10.1093/emboj/20.7.1774

Burgel, P. R., Bellis, G., Olesen, H. V., Viviani, L., Zolin, A., Blasi, F., Elborn, J. S., Bell, S., Castellani, C., Dembski, B., Drevinek, P., Heijerman, H., Innes, A., Lindblad, A., Madge, S., De Rijcke, K., \& Solé, A. (2015). Future trends in cystic fibrosis demography in 34 European countries. European Respiratory Journal , 46 (1), 133-141. https://doi.org/10.1183/09031936.00196314

Chaudary, N. (2018). Triplet CFTR modulators: Future prospects for treatment of cystic fibrosis. In Therapeutics and Clinical Risk Management (Vol. 14, pp. 2375-2383). Dove Medical Press Ltd. https://doi.org/10.2147/TCRM.S147164

Choi, J. Y., Muallem, D., Kiselyov, K., Lee, M. G., Thomas, P. J., \& Muallem, S. (2001). Aberrant CFTRdependent HCO3- transport in mutations associated with cystic fibrosis. Nature , 410 (6824), 94-97. https://doi.org/10.1038/35065099

Clancy, J. P. (2018). Rapid therapeutic advances in CFTR modulator science. Pediatric Pulmonology, 53 (S3), S4-S11. https://doi.org/10.1002/ppul.24157

Cuppens, H., Lin, W., Jaspers, M., Costes, B., Teng, H., Vankeerberghen, A., Jorissen, M., Droogmans, G., Reynaert, I., Goosens, M., Nilius, B., \& Cassiman, J. J. (1998). Polyvariant mutant cystic fibrosis transmembrane conductance regulator genes: The polymorphic (TG)m locus explains the partial penetran- 
ce of the T5 polymorphism as a disease mutation. Journal of Clinical Investigation , 101 (2), 487-496. https://doi.org/10.1172/JCI639

Dal Mas, A., Fortugno, P., Donadon, I., Levati, L., Castiglia, D., \& Pagani, F. (2015). Exon-specific U1s correct SPINK5 exon 11 skipping caused by a synonymous substitution that affects a bifunctional splicing regulatory element. Human Mutation , 36 (5), 504-512. https://doi.org/10.1002/humu.22762

Dal Mas, A., Rogalska, M. E., Bussani, E., \& Pagani, F. (2015). Improvement of SMN2 pre-mRNA processing mediated by exon-specific U1 small nuclear RNA. American Journal of Human Genetics ,96 (1), 93-103. https://doi.org/10.1016/j.ajhg.2014.12.009

De Boeck, K., \& Amaral, M. D. (2016). Progress in therapies for cystic fibrosis. In The Lancet Respiratory Medicine (Vol. 4, Issue 8, pp. 662-674). Lancet Publishing Group. https://doi.org/10.1016/S22132600(16)00023-0

Dekkers, J. F., Berkers, G., Kruisselbrink, E., Vonk, A., De Jonge, H. R., Janssens, H. M., Bronsveld, I., Van De Graaf, E. A., Nieuwenhuis, E. E. S., Houwen, R. H. J., Vleggaar, F. P., Escher, J. C., De Rijke, Y. B., Majoor, C. J., Heijerman, H. G. M., De Winter-De Groot, K. M., Clevers, H., Van Der Ent, C. K., \& Beekman, J. M. (2016). Characterizing responses to CFTR-modulating drugs using rectal organoids derived from subjects with cystic fibrosis. Science Translational Medicine , 8 (344), 344ra84344ra84. https://doi.org/10.1126/scitranslmed.aad8278

Dekkers, J. F., Wiegerinck, C. L., De Jonge, H. R., Bronsveld, I., Janssens, H. M., De Winter-De Groot, K. M., Brandsma, A. M., De Jong, N. W. M., Bijvelds, M. J. C., Scholte, B. J., Nieuwenhuis, E. E. S., Van Den Brink, S., Clevers, H., Van Der Ent, C. K., Middendorp, S., \& Beekman, J. M. (2013). A functional CFTR assay using primary cystic fibrosis intestinal organoids. Nature Medicine , 19 (7), 939-945. https://doi.org/10.1038/nm.3201

Donadon, I., Bussani, E., Riccardi, F., Licastro, D., Romano, G., Pianigiani, G., Pinotti, M., Konstantinova, P., Evers, M., Lin, S., Rüegg, M. A., \& Pagani, F. (2019). Rescue of spinal muscular atrophy mouse models with AAV9-Exon-specific U1 snRNA. Nucleic Acids Research , 47 (14), 7618-7632. https://doi.org/10.1093/nar/gkz469

Donadon, I., Pinotti, M., Rajkowska, K., Pianigiani, G., Barbon, E., Morini, E., Motaln, H., Rogelj, B., Mingozzi, F., Slaugenhaupt, S. A., \& Pagani, F. (2018). Exon-specific U1 snRNAs improve ELP1 exon 20 definition and rescue ELP1 protein expression in a familial dysautonomia mouse model. Human Molecular Genetics , 27 (14), 2466-2476. https://doi.org/10.1093/hmg/ddy151

Du, Q., Li, Z., Pan, Y., Liu, X., Pan, B., \& Wu, B. (2014). The CFTR M470V, intron 8 poly-T, and 8 TGrepeats detection in Chinese males with congenital bilateral absence of the vas deferens. BioMed Research International , 2014, 689185. https://doi.org/10.1155/2014/689185

Dujardin, G., Commandeur, D., Le Jossic-Corcos, C., Ferec, C., \& Corcos, L. (2011). Splicing defects in the CFTR gene: Minigene analysis of two mutations, $1811+1 \mathrm{G}>\mathrm{C}$ and $1898+3 \mathrm{~A}>\mathrm{G}$. Journal of Cystic Fibrosis , 10 (3), 212-216. https://doi.org/10.1016/j.jcf.2010.12.008

Fajac, I., \& Wainwright, C. E. (2017). New treatments targeting the basic defects in cystic fibrosis. In Presse Medicale (Vol. 46, Issue 6P2, pp. e165-e175). Elsevier Masson SAS. https://doi.org/10.1016/j.lpm.2017.01.024

Farinha, C. M., \& Matos, P. (2016). Repairing the basic defect in cystic fibrosis - One approach is not enough. In FEBS Journal(Vol. 283, Issue 2, pp. 246-264). Blackwell Publishing Ltd. https://doi.org/10.1111/febs.13531

Farrell, P. M., White, T. B., Ren, C. L., Hempstead, S. E., Accurso, F., Derichs, N., Howenstine, M., McColley, S. A., Rock, M., Rosenfeld, M., Sermet-Gaudelus, I., Southern, K. W., Marshall, B. C., \& Sosnay, 
P. R. (2017). Diagnosis of Cystic Fibrosis: Consensus Guidelines from the Cystic Fibrosis Foundation. Journal of Pediatrics , 181, S4-S15.e1. https://doi.org/10.1016/j.jpeds.2016.09.064

Gees, M., Musch, S., Van Der Plas, S., Wesse, A. S., Vandevelde, A., Verdonck, K., Mammoliti, O., Hwang, T. C., Sonck, K., Stouten, P., Swensen, A. M., Jans, M., Van Der Schueren, J., Nelles, L., Andrews, M., \& Conrath, K. (2018). Identification and characterization of novel CFTR potentiators. Frontiers in Pharmacology , 9 (OCT). https://doi.org/10.3389/fphar.2018.01221

Gentzsch, M., \& Mall, M. A. (2018). Ion Channel Modulators in Cystic Fibrosis. In Chest (Vol. 154, Issue 2, pp. 383-393). Elsevier Inc. https://doi.org/10.1016/j.chest.2018.04.036

Giuliano, K. A., Wachi, S., Drew, L., Dukovski, D., Green, O., Bastos, C., Cullen, M. D., Hauck, S., Tait, B. D., Munoz, B., Lee, P. S., \& Miller, J. P. (2018). Use of a High-Throughput Phenotypic Screening Strategy to Identify Amplifiers, a Novel Pharmacological Class of Small Molecules That Exhibit Functional Synergy with Potentiators and Correctors. SLAS Discovery, 23 (2), 111-121. https://doi.org/10.1177/2472555217729790

Gregory, R. J., Cheng, S. H., Rich, D. P., Marshall, J., Paul, S., Hehir, K., Ostedgaard, L., Klinger, K. W., Welsh, M. J., \& Smith, A. E. (1990). Expression and characterization of the cystic fibrosis transmembrane conductance regulator. Nature , 347 (6291), 382-386. https://doi.org/10.1038/347382a0

Groman, J. D., Hefferon, T. W., Casals, T., Bassas, L., Estivill, X., Des Georges, M., Guittard, C., Koudova, M., Fallin, M. D., Nemeth, K., Fekete, G., Kadasi, L., Friedman, K., Schwarz, M., Bombieri, C., Pignatti, P. F., Kanavakis, E., Tzetis, M., Schwartz, M., .. Cutting, G. R. (2004). Variation in a Repeat Sequence Determines Whether a Common Variant of the Cystic Fibrosis Transmembrane Conductance Regulator Gene Is Pathogenic or Benign. American Journal of Human Genetics , 74 (1), 176-179. https://doi.org/10.1086/381001

Guimbellot, J., Sharma, J., \& Rowe, S. M. (2017). Toward inclusive therapy with CFTR modulators: Progress and challenges. InPediatric Pulmonology (Vol. 52, Issue S48, pp. S4-S14). https://doi.org/10.1002/ppul.23773

Hoy, S. M. (2019). Elexacaftor/Ivacaftor/Tezacaftor: First Approval. InDrugs (Vol. 79, Issue 18, pp. 20012007). Adis. https://doi.org/10.1007/s40265-019-01233-7

Igreja, S., Clarke, L. A., Botelho, H. M., Marques, L., \& Amaral, M. D. (2015). Correction of a cystic fibrosis splicing mutation by antisense oligonucleotides. In Human Mutation (Vol. 37, Issue 2, pp. 209-215). https://doi.org/10.1002/humu.22931

Kosorok, M. R., Wei, W. H., \& Farrell, P. M. (1996). The incidence of cystic fibrosis. Statistics in Medicine , 15 (5), 449-462. https://doi.org/10.1002/(SICI)1097-0258(19960315)15:5<449::AID-SIM173>3.0.CO;2-X

Liang, F., Shang, H., Jordan, N. J., Wong, E., Mercadante, D., Saltz, J., Mahiou, J., Bihler, H. J., \& Mense, M. (2017). High-Throughput Screening for Readthrough Modulators of CFTR PTC Mutations. SLAS Technology, 22 (3), 315-324. https://doi.org/10.1177/2472630317692561

Marcorelles, P., Friocourt, G., Uguen, A., Ledé, F., Férec, C., \& Laquerrière, A. (2014). Cystic Fibrosis Transmembrane Conductance Regulator Protein (CFTR) Expression in the Developing Human Brain: Comparative Immunohistochemical Study between Patients with Normal and Mutated CFTR. Journal of Histochemistry and Cytochemistry ,62 (11), 791-801. https://doi.org/10.1369/0022155414546190

Mattioli, C., Pianigiani, G., De Rocco, D., Bianco, A. M. R., Cappelli, E., Savoia, A., \& Pagani, F. (2014). Unusual splice site mutations disrupt FANCA exon 8 definition. Biochimica et Biophysica Acta - Molecular Basis of Disease , 1842 (7), 1052-1058. https://doi.org/10.1016/j.bbadis.2014.03.014

Merkert, S., Schubert, M., Olmer, R., Engels, L., Radetzki, S., Veltman, M., Scholte, B. J., Zöllner, J., Pedemonte, N., Galietta, L. J. V., von Kries, J. P., \& Martin, U. (2019). High-Throughput Screening for Modulators of CFTR Activity Based on Genetically Engineered Cystic Fibrosis Disease-Specific iPSCs. Stem Cell Reports , 12 (6), 1389-1403. https://doi.org/10.1016/j.stemcr.2019.04.014 
Nizzardo, M., Simone, C., Dametti, S., Salani, S., Ulzi, G., Pagliarani, S., Rizzo, F., Frattini, E., Pagani, F., Bresolin, N., Comi, G., \& Corti, S. (2015). Spinal muscular atrophy phenotype is ameliorated in human motor neurons by SMN increase via different novel RNA therapeutic approaches. Scientific Reports , 5 . https://doi.org/10.1038/srep11746

Ohkubo, A., Kondo, Y., Suzuki, M., Kobayashi, H., Kanamori, T., Masaki, Y., Seio, K., Nagai, K., \& Sekine, M. (2013). Chemical synthesis of U1 snRNA derivatives. Organic Letters , 15 (17), 4386-4389. https://doi.org/10.1021/ol401917r

Ontalus, V., Cobianu, C., Vasiliu, F., \& Parlog, C. (1996). Crystallization behaviour and phase coexistence at morphotrophic phase boundaries in PZT thin films prepared by sol-gel processing. Journal of Materials Science , 31 (14), 3639-3642. https://doi.org/10.1007/BF00352771

Pagani, F., Buratti, E., Stuani, C., \& Baralle, F. E. (2003). Missense, nonsense, and neutral mutations define juxtaposed regulatory elements of splicing in cystic fibrosis transmembrane regulator exon 9.Journal of Biological Chemistry, 278 (29), 26580-26588. https://doi.org/10.1074/jbc.M212813200

Pagani, F., Buratti, E., Stuani, C., Bendix, R., Dörk, T., \& Baralle, F. E. (2002). A new type of mutation causes a splicing defect in ATM.Nature Genetics , 30 (4), 426-429. https://doi.org/10.1038/ng858

Pagani, F., Buratti, E., Stuani, C., Romano, M., Zuccato, E., Niksic, M., Giglio, L., Faraguna, D., \& Baralle, F. E. (2000). Splicing factors induce cystic fibrosis transmembrane regulator exon 9 skipping through a nonevolutionary conserved intronic element. Journal of Biological Chemistry , 275 (28), 21041-21047. https://doi.org/10.1074/jbc.M910165199

Palomaki, G. E., Fitzsimmons, S. C., \& Haddow, J. E. (2004). Clinical sensitivity of prenatal screening for cystic fibrosis via CFTR carrier testing in a United States panethnic population. Genetics in Medicine, 6 (5), 405-414. https://doi.org/10.1097/01.GIM.0000139505.06194.39

Paranjape, S. M., \& Mogayzel, P. J. (2018). Cystic fibrosis in the era of precision medicine. In Paediatric Respiratory Reviews (Vol. 25, pp. 64-72). W.B. Saunders Ltd. https://doi.org/10.1016/j.prrv.2017.03.001

Pereira, S. V. N., Ribeiro, J. D., Ribeiro, A. F., Bertuzzo, C. S., \& Marson, F. A. L. (2019). Novel, rare and common pathogenic variants in the CFTR gene screened by high-throughput sequencing technology and predicted by in silico tools. Scientific Reports , 9 (1). https://doi.org/10.1038/s41598-019-42404-6

Phuan, P. W., Son, J. H., Tan, J. A., Li, C., Musante, I., Zlock, L., Nielson, D. W., Finkbeiner, W. E., Kurth, M. J., Galietta, L. J., Haggie, P. M., \& Verkman, A. S. (2018). Combination potentiator ('copotentiator') therapy for CF caused by CFTR mutants, including N1303K, that are poorly responsive to single potentiators. Journal of Cystic Fibrosis , 17 (5), 595-606. https://doi.org/10.1016/j.jcf.2018.05.010

Pranke, I., Golec, A., Hinzpeter, A., Edelman, A., \& Sermet-Gaudelus, I. (2019). Emerging therapeutic approaches for cystic fibrosis. From gene editing to personalized medicine. In Frontiers in Pharmacology (Vol. 10, Issue FEB). Frontiers Media S.A. https://doi.org/10.3389/fphar.2019.00121

Pranke, I. M., Hatton, A., Simonin, J., Jais, J. P., Le Pimpec-Barthes, F., Carsin, A., Bonnette, P., Fayon, M., Stremler-Le Bel, N., Grenet, D., Thumerel, M., Mazenq, J., Urbach, V., Mesbahi, M., Girodon-Boulandet, E., Hinzpeter, A., Edelman, A., \& Sermet-Gaudelus, I. (2017). Correction of CFTR function in nasal epithelial cells from cystic fibrosis patients predicts improvement of respiratory function by CFTR modulators. Scientific Reports , 7 (1), 1-11. https://doi.org/10.1038/s41598-017-07504-1

Rogalska, M. E., Tajnik, M., Licastro, D., Bussani, E., Camparini, L., Mattioli, C., \& Pagani, F. (2016). Therapeutic activity of modified U1 core spliceosomal particles. Nature Communications , 7, 1-13. https://doi.org/10.1038/ncomms11168

Roomans, G. M. (2014). Pharmacological treatment of the basic defect in cystic fibrosis. Cell Biology International , 38 (11), 1244-1246. https://doi.org/10.1002/cbin.10312 
Sato, T., Stange, D. E., Ferrante, M., Vries, R. G. J., Van Es, J. H., Van Den Brink, S., Van Houdt, W. J., Pronk, A., Van Gorp, J., Siersema, P. D., \& Clevers, H. (2011). Long-term expansion of epithelial organoids from human colon, adenoma, adenocarcinoma, and Barrett's epithelium. Gastroenterology , 141 (5), 1762-1772. https://doi.org/10.1053/j.gastro.2011.07.050

Sato, T., Vries, R. G., Snippert, H. J., Van De Wetering, M., Barker, N., Stange, D. E., Van Es, J. H., Abo, A., Kujala, P., Peters, P. J., \& Clevers, H. (2009). Single Lgr5 stem cells build crypt-villus structures in vitro without a mesenchymal niche. Nature , 459 (7244), 262-265. https://doi.org/10.1038/nature07935

Schram, C. A. (2012). Atypical cystic fibrosis: identification in the primary care setting. Canadian Family Physician Medecin de Famille Canadien , 58 (12), 1341-1345, e699-704. http://www.ncbi.nlm.nih.gov/pubmed/23242890

Strug, L. J., Stephenson, A. L., Panjwani, N., \& Harris, A. (2018). Recent advances in developing therapeutics for cystic fibrosis.Human Molecular Genetics , 27 (R2), R173-R186. https://doi.org/10.1093/hmg/ddy188

Tajnik, M., Rogalska, M. E., Bussani, E., Barbon, E., Balestra, D., Pinotti, M., \& Pagani, F. (2016). Molecular Basis and Therapeutic Strategies to Rescue Factor IX Variants That Affect Splicing and Protein Function. PLoS Genetics , 12 (5), 1-16. https://doi.org/10.1371/journal.pgen.1006082

Tang, Y.-Y., Hölzel, B. K., \& Posner, M. I. (2015). The neuroscience of mindfulness meditation. Nature Reviews Neuroscience ,16 (4), 213-225. https://doi.org/10.1038/nrn3916

Van Goor, F., Hadida, S., Grootenhuis, P. D. J., Burton, B., Cao, D., Neuberger, T., Turnbull, A., Singh, A., Joubran, J., Hazlewood, A., Zhou, J., McCartney, J., Arumugam, V., Decker, C., Yang, J., Young, C., Olson, E. R., Wine, J. J., Frizzell, R. A., ... Negulescu, P. (2009). Rescue of CF airway epithelial cell function in vitro by a CFTR potentiator, VX-770. Proceedings of the National Academy of Sciences of the United States of America , 106 (44), 18825-18830. https://doi.org/10.1073/pnas.0904709106

Wang, P., Lin, Z., Su, X., \& Tang, Z. (2017). Application of Au based nanomaterials in analytical science. In Nano Today (Vol. 12, Issue 13, pp. 64-97). https://doi.org/10.1016/j.nantod.2016.12.009

Wu, H. xia, Zhu, M., Xiong, X. feng, Wei, J., Zhuo, K. quan, \& Cheng, D. yun. (2019). Efficacy and Safety of CFTR Corrector and Potentiator Combination Therapy in Patients with Cystic Fibrosis for the F508del-CFTR Homozygous Mutation: A Systematic Review and Meta-analysis.Advances in Therapy , 36 (2), 451-461. https://doi.org/10.1007/s12325-018-0860-4

Yeh, H. I., Sohma, Y., Conrath, K., \& Hwang, T. C. (2017). A common mechanism for CFTR potentiators. Journal of General Physiology ,149 (12), 1105-1118. https://doi.org/10.1085/jgp.201711886

Zielenski, J. (2000). Genotype and phenotype in cystic fibrosis. InRespiration (Vol. 67, Issue 2, pp. 117-133). S. Karger AG. https://doi.org/10.1159/000029497

Zuccato, E., Buratti, E., Stuani, C., Baralle, F. E., \& Pagani, F. (2004). An Intronic Polypyrimidinerich Element Downstream of the Donor Site Modulates Cystic Fibrosis Transmembrane Conductance Regulator Exon 9 Alternative Splicing. Journal of Biological Chemistry ,279 (17), 16980-16988. https://doi.org/10.1074/jbc.M313439200

Zychlinski, D., Erkelenz, S., Melhorn, V., Baum, C., Schaal, H., \& Bohne, J. (2009). Limited complementarity between U1 snRNA and a retroviral 5' splice site permits its attenuation via RNA secondary structure. Nucleic Acids Research , 37 (22), 7429-7440. https://doi.org/10.1093/nar/gkp694

\section{Figure legends}

Figure 1: Exon Specific U1s rescue splicing defects that cause skipping of CFTR exons. Panels A), B), C), D) and E) show minigene splicing assay for CFTR exons 5, 9, 13, 16 and 18, respectively. The indicated WT and mutant minigene variants were transfected in HeLa cells alone or co-transfected with the indicated 
ExSpeU1s. In each panel, the upper part shows a schematic representation of CFTR exons, mutants and ExSpeU1s; the intermediate figure shows the agarose gel and the lower panel the percentage of exon inclusion. Identity of the exon inclusion and skipping band are indicated. In B), the middle band corresponds to the activation of a previously described cryptic splice site (Buratti et al., 2001).

Figure 2: Exon Specific U1s rescue CFTR protein levels in exon 13 and exon 16 splicing mutations. For each panel the upper part shows schematic representation of CFTR exons along with mutants and position of the ExSpeU1s, the intermediate figure shows the Western Blot analysis and the lower panel the percentage of CFTR protein normalized over Tubulin. Data are expressed as means +- SD of three independent experiments done in duplicate. A) Western Blot analysis of exon 13 with total CFTR cDNA, CFTR cDNA without Exon13, CFTR cDNA flanked by partial sequence of intron 12 and 13, 1863C > T, 1898+3A>G mutants and ExSpeU1. B) Western Blot analysis of exon 16 with WT, 2789+5G>A mutant and ExSpeU1s.

Figure 3: The Intronic Splicing Silencer (ISS) in CFTR intron 13 overlaps with the U1ex13-11 binding site. A) Effect of consecutive intron 13 deletions on CFTR exon 13 splicing in minigene assay in WT, $1863 \mathrm{C}>\mathrm{T}$ and $1898+3 \mathrm{~A}>\mathrm{G}$ variants. The upper panel indicates the exon13-intron 13 region along with the position of intronic deletions and mutants. Position +1 is the first base of the intron. The intermediate panel is a representative agarose gel of the splicing assay and the lower graph shows of the percentage of exon inclusion expressed as the means +- SD of three independent experiments done in duplicate. B) Predicted RNA secondary structure of CFTR exon 13-intron 13 region (RNA m-fold analysis). The ISS is indicated by a black line. Exonic sequences are in the grey box and intronic sequences are in lowercase. Position +1 is the first base of the intron. C) Fine mapping of the ISS in WT context. The upper panel shows the sequence of the exon13-intron 13 junction along with the position of the ISS point mutations from position 12 to 23. Position +1 is the first base of the intron. The intermediate panel is a representative agarose gel of the splicing assay of the indicated single or double mutant minigenes. The lower graph shows the percentage of exon inclusion expressed as the means +- SD of three independent experiments done in duplicate. D) The relationship between percentage of exon 13 inclusion (y axis) and changes in estimated $\Delta \mathrm{G}$ (x axis) fitted in sigmoidal function.

\section{Hosted file}

Table_1.docx available at https://authorea.com/users/341804/articles/468685-rescue-ofcommon-exon-skipping-mutations-in-cystic-fibrosis-with-modified-u1-snrnas 
A)

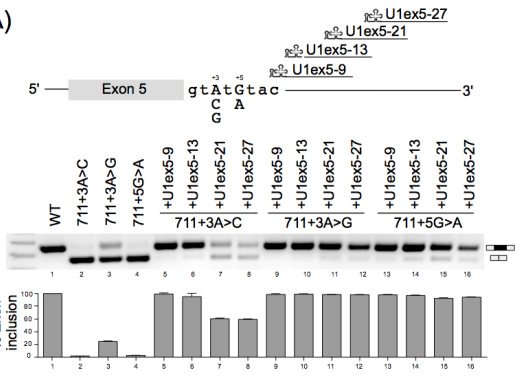

B)
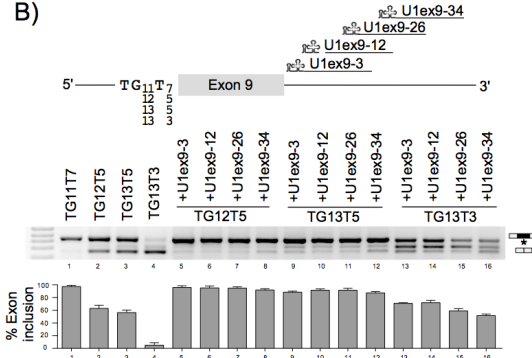

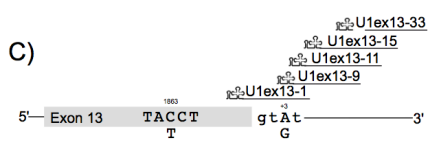

D)

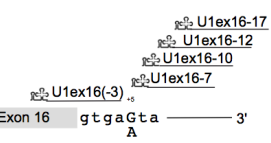

E)
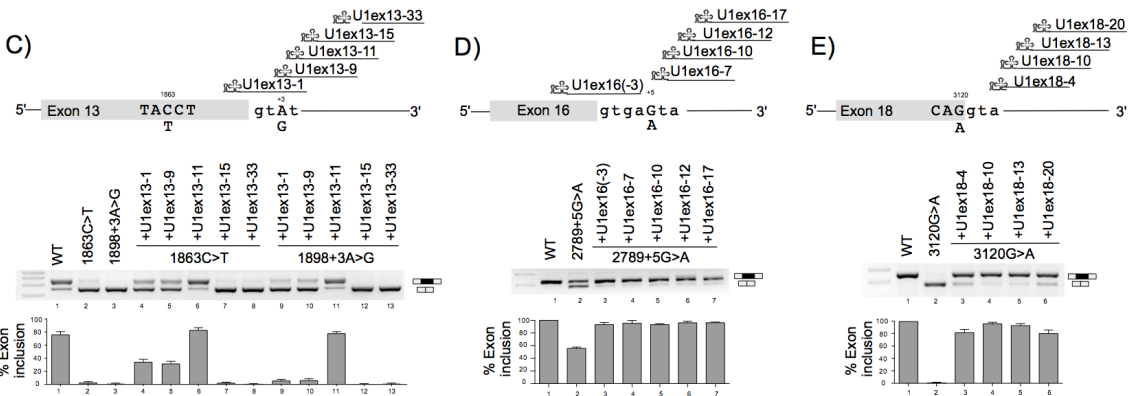

A)

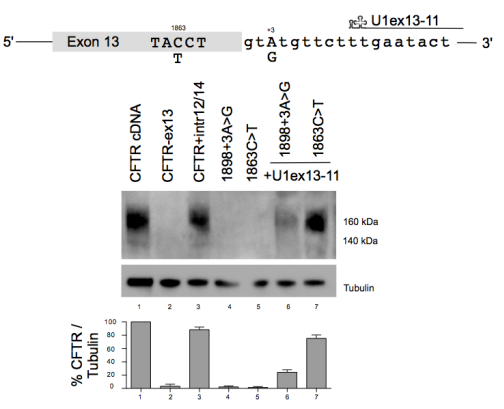

B)

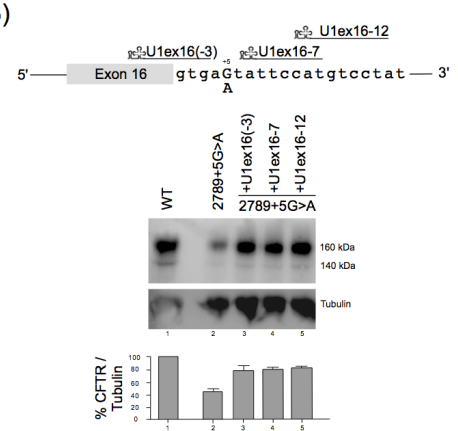




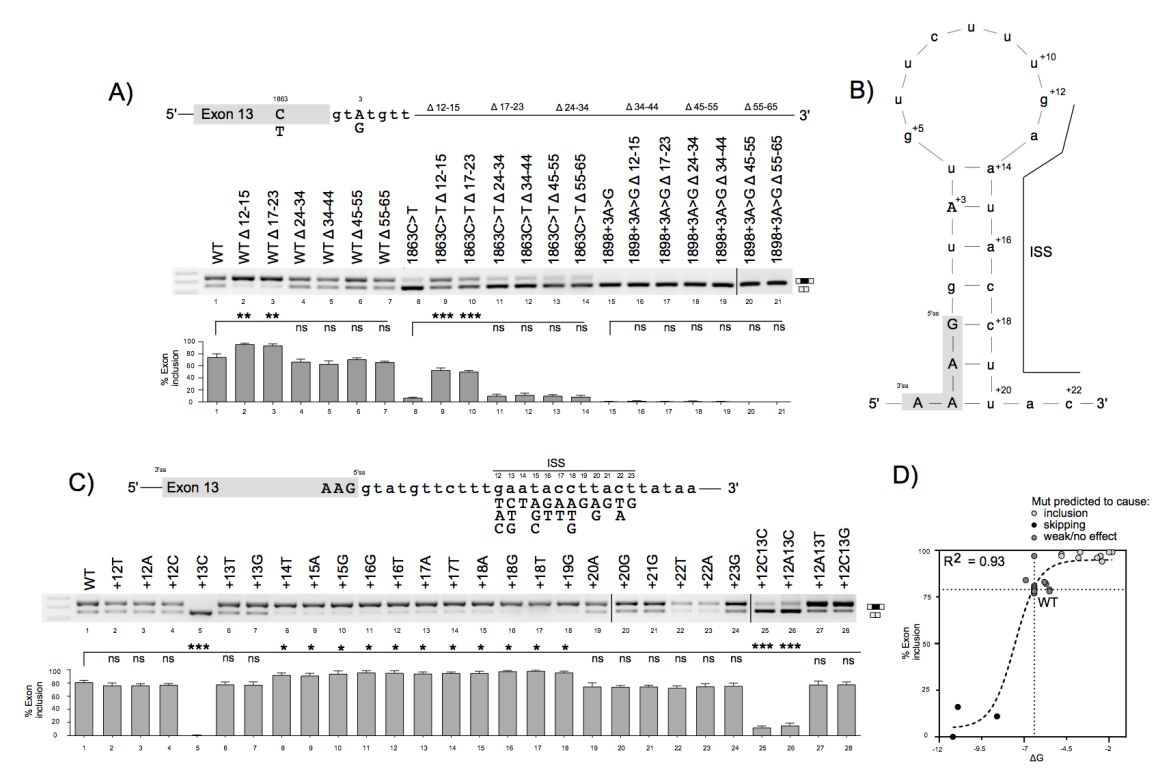

\section{Hosted file}

Table_S1.docx available at https://authorea.com/users/341804/articles/468685-rescue-ofcommon-exon-skipping-mutations-in-cystic-fibrosis-with-modified-u1-snrnas 